\title{
Six DOF Motion Estimation for Teleoperated Flexible Endoscopes Using Optical Flow: A Comparative Study
}

\author{
Charreau S. Bell, Gustavo A. Puerto, Gian-Luca Mariottini, and Pietro Valdastri
}

\begin{abstract}
Colorectal cancer is one of the leading causes of cancer-related deaths worldwide, although it can be effectively treated if detected early. Teleoperated flexible endoscopes are an emerging technology to promote participation in these preventive screenings. Real-time pose estimation is therefore essential to enable feedback to the robotic endoscope's control system. Vision-based endoscope localization approaches are a promising avenue, since they do not require extra sensors on board the endoscopes. In this work, we compare several stateof-the-art algorithms for computing the image motion (optical flow), which is then used with a supervised learning strategy to provide an accurate estimate of the 6 degree of freedom endoscope motion. The method is validated using a robotically actuated endoscope in a human colon simulator, and represents a preliminary effort towards testing with clinical video data.
\end{abstract}

\section{INTRODUCTION}

Colorectal cancer is the fourth leading cause of cancer mortality in the world, with more than $63 \%$ of these deaths occurring in developed nations [1], [2]. Unlike many other types of cancer, colorectal cancer can almost always be prevented by regular screenings for patients over 50 or with a family history of colon cancer. By detection at an early stage, the prognosis for survival is $90 \%$, whereas if detected too late, it decreases to $5 \%$ [3].

The traditional method for colorectal cancer screening is through colonoscopy, a procedure in which a $1.5 \mathrm{~m}$ flexible endoscope is inserted into the colon for the detection and removal of lesions and polyps. Colonoscopy is an outpatient procedure, is performed under sedation, and only takes about 30 minutes. Despite these favorable conditions and the clear advantages of compliance with the recommendation for cancer screening, nearly one-third of the population at risk avoid the procedure [4].

In an effort to encourage patient participation and increase the polyp detection rate, fully- and semi-automatic robotic endoscopes are emerging in the field [3], [5]-[8]. This approach focuses the doctor's attention on polyp detection, and reduces the physically demanding maneuvering of the endoscope, which requires years of training for proficiency.

The compliancy of colon tissue and its variability among patients presents a challenge for creating an accurate model

C.S. Bell and P. Valdastri are with the Department of Mechanical Engineering, Vanderbilt University, 506 Olin Hall, Nashville, TN 37235, USA

Email:charreau.s.belldvanderbilt.edu

p.valdastri@vanderbilt.edu

G. Puerto and G.L. Mariottini are with the Department of Compute Science and Engineering, University of Texas at Arlington, 416 Yates Street, Arlington, TX 76019, USA.

Email:gustavo.puerto@mavs.uta.edu

gianluca.mariottini@uta.edu of the colon for open-loop control for robotic endoscopes. Instead, closed-loop control, which is effective for disturbance rejection and error minimization, can decrease the difference between the target actuation pose versus the actual reached pose of the endoscopic camera head.

Real-time pose detection is therefore essential to enabling feedback to the robotic endoscope's control system. Magnetic tracking is a reliable method for localization; however, this can possibly increase the endoscope size or occupy the tool channel. Furthermore, the accuracy and reliability of a magnetic tracker can be potentially compromised by interfering magnetic fields from the permanent magnets of emerging teleoperable platforms that are being pursued commercially and by research labs worldwide [9]-[12].

Image processing provides a valid alternative avenue for localization. One popular method in medicine is through global localization by means of image registration; however, because of the large deformations of the soft colon body during a colonoscopy, global localization is ineffective in practice. Optical flow techniques avoid this limitation, as they provide information about the frame-to-frame changes to compute the motion of the endoscope tip. Such a localization scheme that neither increases the size of the endoscope nor interferes with the robotic platform, but produces reliable and accurate pose estimates, is a favorable method for providing pose feedback for teleoperated flexible endoscopes.

\section{A. Related Work}

Usage of the endoscopic camera stream has mainly focused on locating gastrointestinal structural landmarks or lesions. These large-scale features include the lumen [13], [14] and haustral folds [15]-[17]. The usage of machine learning and artificial intelligence methods for navigation within the colon has also been limited to detecting gastrointestinal structural landmarks via pattern recognition classifiers [18], and rule-based systems using fuzzy logic [15].

Motion estimation and navigation based on image motion analysis for navigation have been successfully used for other applications, including mobile robot and vehicle navigation. Common techniques include optical flow, and modeling of the camera [19], although scale cannot be recovered by these methods alone. Within gastrointestinal endoscopy, navigation has been achieved by adjusting the current heading towards the lumen center in each control loop by employing the spherical camera model [6]. Focus of expansion and optical flow approaches have also been successfully employed on virtual colonoscopy and other real endoscopic image sets [7], [20], [21]. However, algorithm performance on computer- 
generated datasets can differ significantly from a colon simulator or human colon [6], and none of these approaches are able to provide quantitative information about the full 6 degree of freedom (DOF) change of pose of the endoscope tip. Additionally, many of these methods assume that a focusof-expansion can be detected in the image, which might not happen in the frequent case of rotational motions of the endoscope.

\section{B. Original Contribution and Organization}

The original contribution of this paper is to compare the effects of several state-of-the-art optical flow estimation algorithms on their capability to best describe the movement between images of the colon typically observed during a colonoscopy. The efficacy of these optical flow measurements resulting from each of these methods is measured by the accuracy of a supervised learning localization strategy that maps these image variations to $6 \mathrm{DOF}$ changes in pose of the endoscope.

Applying artificial neural networks (ANN) to derive the change in pose of robotic endoscopes has been proposed [22]. In this study, different sources of illumination (white light and narrow band) and image partitioning (grid-based and lumen-centered) were compared to investigate the combination providing the strongest features to drive the ANN. A standard Lucas-Kanade (LK) method was adopted to compute the sparse optical flow. In this paper, we build upon this previous work by providing an extensive comparison of several of the most important optical flow computation methods.

The paper is organized as follows: Sect. II presents an overview of the optical flow methods adopted in our work, together with a description of their major advantages and disadvantages. Sect. III describes the supervised-learning localization strategy, while Sect. IV presents both the experimental setup and the results of the comparison between each optical flow method. Finally, Sect. V highlights the major conclusions and describes future work.

\section{Overview of OpticAl Flow Computation}

In this section, we present an overall description of the state-of-the-art algorithms we adopted for the computation of the image motion (optical flow) across consecutive frames of an endoscopic video. Since a comparison between all the optical flow algorithms designed over the past decades is unfeasible in this conference paper, we decided to focus on a subset of representative methods. In particular, we selected those methods that are most popular and with important peculiar features, such as invariance to illumination or rapid camera motion. The mathematical and implementation details for each method are outside the scope of this work and can be obtained from the references provided below.

\section{A. Lucas-Kanade (LK) based optical flow}

We adopted the Lucas-Kanade method [23] for computing the optical flow between two frames of an endoscopic video. LK estimates the image motion of image templates across

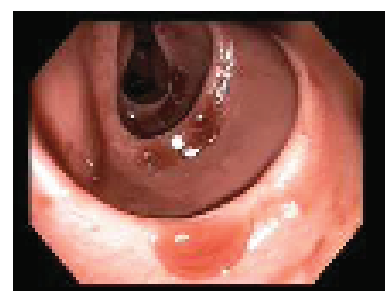

(a) $I_{t-\Delta t}$

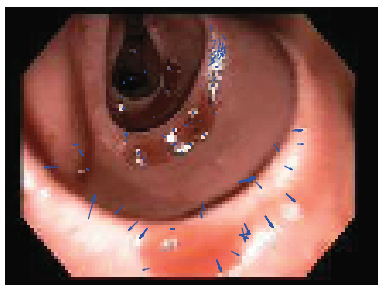

(c) LK

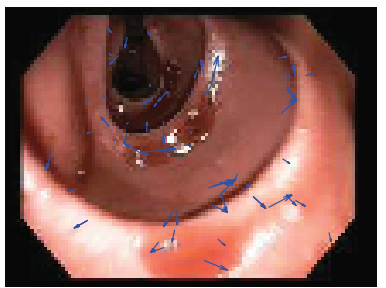

(e) SIFT

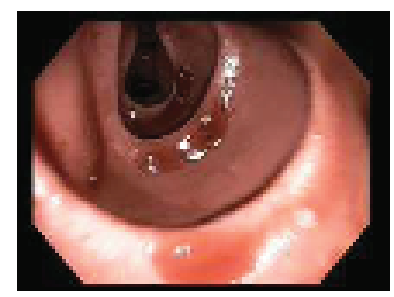

(b) $I_{t}$

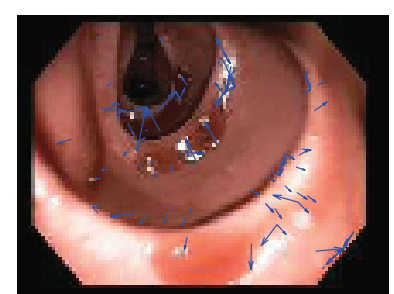

(d) SURF

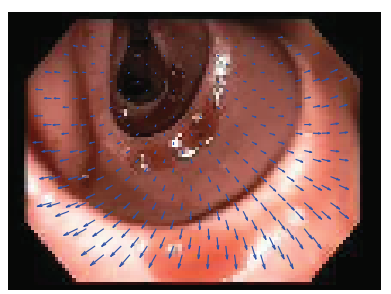

(f) dHMA
Fig. 1. Representative Optical Flows: (a)-(b) Frames $I_{t-\Delta t}$ and $I_{t}$; (c) Lucas-Kanade (LK); (d) Scale-invariant (SURF); (e) Scale-invariant (SIFT); (f) Dense Hierarchical Multi-Affine (dHMA).

two consecutive frames, $I_{t-\Delta t}$ and $I_{t}$ (cf., Fig. 1(a)-1(b)), of an endoscopic video. As commonly done in the literature, we centered each template at a Shi-Tomasi feature [24]. We adopted a pyramidal multi-resolution implementation of the LK method [25] that provides a more reliable estimation when compared to traditional LK implementations.

Figure 1(c) illustrates an example of the optical flow vectors (blue) computed by LK. Each flow vector is centered at a Shi-Tomasi corner. Because of the presence of many textureless areas and image blurs, the resulting LK-based optical flow is usually very sparse, since only few ShiTomasi features are detected in these areas. There are two key assumptions in the LK method: the first one assumes brightness constancy over time of the two frames, and the second assumes small motion of each template across consecutive frames, allowing filtering of noisy flow vectors with very large magnitude (outliers).

\section{B. Scale-Invariant optical flow}

In order to robustly estimate the optical flow in the case of large endoscope motion and changes in illumination, we adopted two scale-invariant features: SIFT (Scale-Invariant Feature Transform) [26] and SURF (Speeded-Up Robust Features). These features are extracted and matched in two consecutive frames to find the optical flow. We decided to focus on these two algorithms because of their proved high- 
repeatability (SIFT), speed (SURF), and because of their good matching and accuracy rates (SURF and SIFT) in many challenging conditions.

The main advantage of these scale-invariant features, when compared to other feature extraction and matching methods, is that a descriptor is computed from the image portion around each keypoint (i.e., an image patch, edge, or corner). The importance of descriptors is that they contain a statistical representation of the image gradients around each local image portion. As such, the descriptor is what makes SIFT and SURF invariant to changes in scale and rotation, and robust to changes in illumination and viewpoint [27].

Figure 1(e) illustrates the optical flow obtained with SIFT descriptors between two sequential frames. SIFT (as well as SURF) can extract optical flow vectors in (small) areas with uniform texture. However, it is possible to still see the presence of a few wrong flow vectors (outliers) caused by the erroneously-matched descriptors.

In our implementation, we tested several keypoint detectors, being FAST keypoint [28] the one that offered better results in this scenario.

\section{Dense Hierarchical Multi-Affine (dHMA) optical flow}

We adopted two dense optical-flow methods that were proved to have a reduced outlier ratio when compared to SIFT and SURF, especially when applied to endoscopic images. In particular, we adopted HMA [29], [30] and dHMA [31]; the latter can (densely) estimate the optical flow vectors over the entire image, and thus cover large textureless areas. Differently from other dense optical-flow methods that take tens of seconds, HMA and dHMA almost runs in real time $(\sim 80 \mathrm{~ms}$ for HMA, and $\sim 1$ sec. for dHMA $)$ in our MATLAB implementation.

Both HMA and dHMA match sparse feature descriptors ${ }^{1}$ while eliminating the majority of outliers by means of a robust hierarchical estimation of multi-affine transformations. dHMA has an additional step in which the resulting inliers are given in input to a Gaussian-Process regression stage [32] that estimates a dense mapping function. This function (or the multi-affine maps from HMA) model the non-rigid motion of a given set of image points over the entire image (ideally, for every pixel).

Figure 1(f) shows the optical flow vectors calculated by dHMA for a pre-defined grid of image points. Since these points do not necessarily coincide with image features (e.g., corners), the resulting optical flow can be computed over large textureless areas, and with few outliers. Compared to the aforementioned optical-flow estimation methods, HMA and dHMA have great accuracy, can eliminate outliers, are automatic, and require very minimal number of tuning parameters from the user. Our previous results [31] experiments showed that dHMA can achieve a slight better accuracy than HMA.

\footnotetext{
${ }^{1}$ In our implementation, HMA and dHMA uses SIFT descriptors.
}

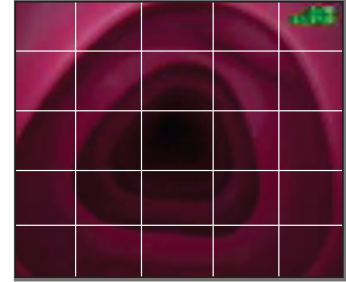

(a)

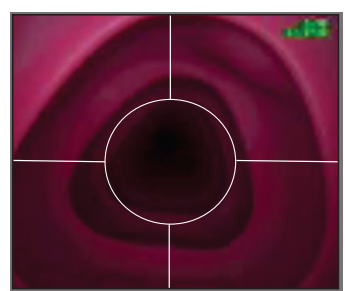

(b)
Fig. 2. Spatial partitioning rules for creating the optical flow descriptors from an optical flow field. (a) Grid-based partitioning. (b) Lumen-centered partitioning.

\section{Flow DESCRIPTORS AND LOCALIZATION STRATEGY}

The proposed method estimates the 6 DOF change in pose of the endoscope camera tip using only optical flow data computed as described in Sect. II. The scene viewed by the camera is assumed to be static between two frames; this is a valid assumption since large-scale motion in the colon is limited to haustral contractions (i.e., motion which moves the contents of the colon forward). These movements occur relatively rarely (once every 30 minutes) [33]. Regardless, specialized control loops using histogram analysis and other heuristics can be implemented to mitigate the effects of sporadic deviations from these assumption.

The optical flow vector field extracted at each time instant over the entire image is combined into a single representative vector, referred to as the optical flow descriptor. We examine two distinct ways of combining the entire flow field at each frame into a single descriptor: a grid-based or a lumencentered partition. These optical flow descriptors, together with a known metric change in pose of the endoscope, compose the training set for a supervised learning method, specifically, an artificial neural network (ANN). Once trained, the ANN provides an associative model of the system for realtime pose feedback; given only the relative motion in the image, the ANN is able to estimate the change in pose of the endoscope tip.

\section{A. Feature Descriptor Generation}

Figure 3 outlines the process for computing the change in pose from the endoscopic camera sequence. At times $t-\Delta t$ and $t$, images are captured from the camera. ${ }^{2}$ These two images are then used to compute the optical flow according to the methods presented in Section II.

The entire optical flow is then used to create a single optical flow descriptor. Two different image partitioning methods were adopted and compared: a standard grid-based partitioning (cf., Fig. 2(a)), and lumen-centered partitioning (cf., Fig. 2(b)).

The grid-based partitioning method divides the image into a $5 \times 5$ grid (i.e., 25 sections) of equal rectangular areas. The $n_{g}$ optical flow vectors inside each region $g$ of the

\footnotetext{
${ }^{2} \mathrm{~A}$ mask was applied in order to only include the effective pixels in the image in the algorithm, and disregard any text present in the image.
} 


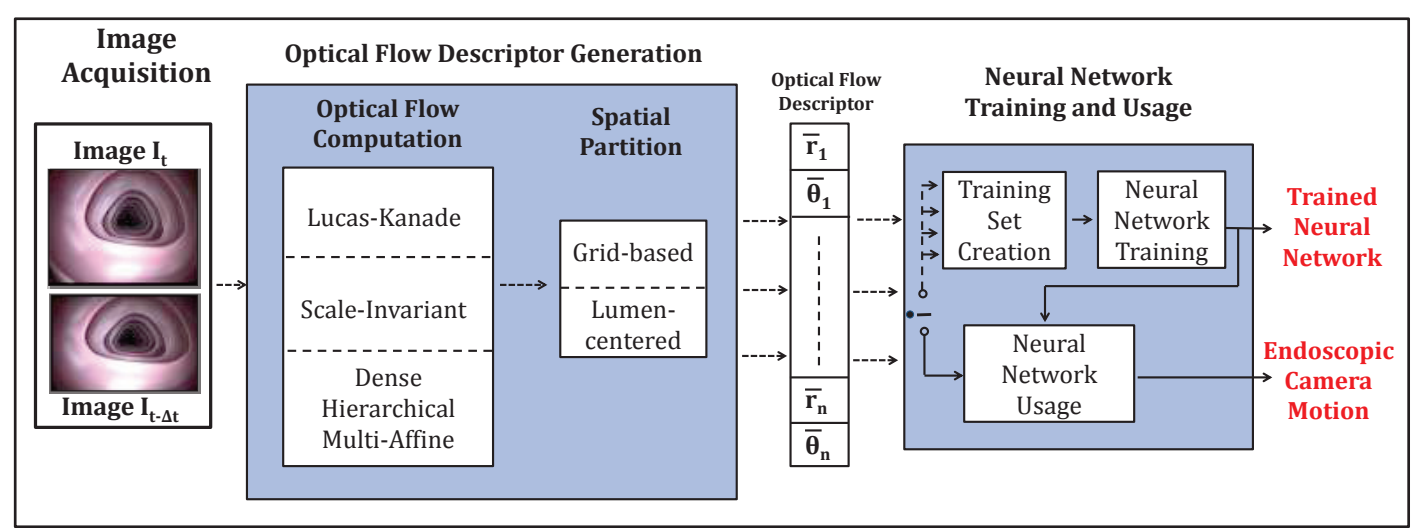

Fig. 3. Flow diagram for the proposed method for comparing methods of optical flow (Lucas-Kanade vs. Scale Invariant vs. Dense Hierarchical MultiAffine) and partitioning (grid-based vs. lumen-centered) and their use with artificial neural networks for pose estimation.

whole grid-based partitioning $G_{G}$ are used to calculate two elements of the flow descriptor as follows:

$$
\bar{r}_{g}=\frac{\sum_{i=1}^{n_{g}} \sqrt{d x_{i}^{2}+d y_{i}^{2}}}{n_{g}}, \quad \bar{\theta}_{g}=\frac{\sum_{i=1}^{n_{g}} \operatorname{atan} 2\left(d y_{i}, d x_{i}\right)}{n_{g}}
$$

where $\bar{r}_{g}$ is the average over all the lengths of optical flow between corresponding features in region $g$, and $\bar{\theta}_{g}$ is the average orientation of the flow-field vectors in region $g$.

The lumen-centered partitioning method utilizes similar descriptors defining 5 regions divided based on the structure of the colon. The first region is computed based on the lumen; the image is histogram equalized and thresholded to extract the darkest portion of the image, corresponding to the lumen. To delimit the other four regions of the colon, the image is segmented vertically and horizontally based on the centroid of the lumen to divide the image into 4 quadrants. The four remaining regions are defined as the portions of these quadrants not including the area defined as the lumen.

Note that before computing the average, a median operator was initially applied to reject possible outliers flow fields (i.e., vectors with an erroneous orientation with respect to the majority). In order to form the feature descriptor vector for the entire image, the two elements $\left(\bar{r}_{g}, \bar{\theta}_{g}\right)$ for each of the grid spaces are concatenated into one single feature descriptor of size 50 (i.e., 2 elements for each grid region).

\section{B. Neural Network Training and Usage}

The obtained optical flow descriptors are then used as the inputs to multi-layer feedforward ANN, which is able to learn complex nonlinear input/output mappings [34]. Levenberg-Marquardt backpropagation [35], [36] was used to adjust the weights of the neural networks (i.e., train the ANN). The topology of each of the neural networks was chosen to be $2 n+1$ hidden layer architecture [37], where $n$ indicates the dimension of the inputs to the neural network (i.e., the number of nodes in the feature vector). This corresponds to neural network architectures $50 \times 101 \times 6$ for grid-based partitioning, and $10 \times 21 \times 6$ for lumen-centered partitioning.

The ANNs were trained by first dividing the training set into 3 subsequent sets: a training set $(85 \%$ of the training data), a validation set (10\% of the training data), and a testing set $(5 \%$ of the data). During training, each example in the training set is forward propagated through the neural network, producing an estimate of the change in endoscope pose. Using the ground truth target data, the mean square error is generated, which is then used to adjust the weights of the ANN. Early stopping was used in order to avoid overtraining the neural networks.

Once a trained neural network is produced, testing of the performance of the neural network proceeds similarly. Each optical flow descriptor in the testing set is forward propagated through the neural network, which then produces an estimate of the relative motion (i.e., the output of the neural network is the change in pose) of the endoscope.

\section{EXPERIMENTAL RESULTS}

In order to test the validity of the approach, a controlled setup was used in order to evaluate the performance of the algorithm in an artificial scenario (a straight endoscope motion in the colon) and by attaching the endoscope to a robot's end effector to precisely record its motion. This was done in order to mimic the most common and likely movement of a teleoperated robotic endoscope.

The performance of the neural networks was then evaluated based on the means, standard deviations, and the evaluation of the overall trajectory of the integrated estimates against the ground truth obtained by the robot's encoders.

\section{A. Validation Benchtop for 1 DOF}

The purpose of this experiment was to test the proposed approach in one DOF and to determine the most effective combination of features/optical flow algorithms with the highest accuracy in estimating the change in pose of the endoscope tip from camera motion. The experimental setup utilizes a Karl Storz endoscope (13803PKS; Germany) rigidly attached to an industrial robotic arm $( \pm 20 \mu \mathrm{m}$ repeatability, RV-6SDL; Mitsubishi Corporation; Japan). The experimental platform is shown in Figure IV.

Using control software written in $\mathrm{C}++$, at each time step, positional commands are sent to the robot using TCP/IP ethernet protocol, which actuates the robot along the optical 


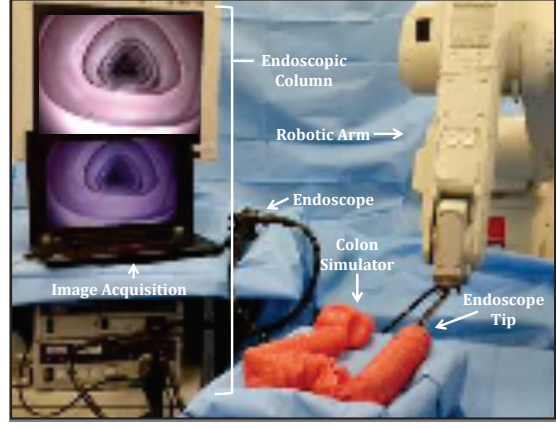

Fig. 4. Experimental setup replication movements of a teleoperated endoscope in a human colon simulator.

axis of the camera (the optical axis of the camera is aligned with the $\mathrm{x}$-axis of the robot). The endoscope moves along a trajectory in the plastic human colon simulator (shown in Figure IV) in increments of $\sim \pm 0.3 \mathrm{~mm}$ from $0.3 \mathrm{~mm}$ to $4 \mathrm{~mm}$ for 20 iterations each. This resulted in a data set of size 260 , corresponding to $549 \mathrm{~mm}$ of total movement. After a one second time delay to avoid oscillation in the image due to the actuation, the resultant frame is captured through a frame grabber (EasyCAP; USB EasyCap Co., China) via SVideo from the Karl Storz endoscopic column, alongside the corresponding position of the robot. This is repeated until the entire trajectory was completed.

Thanks to the rigid connection to the robot, the instantaneous motion can be easily deduced from the robot's encoders with high accuracy. This large dataset is then subsampled into two mutually exclusive groups for the training and testing data, using two-thirds of the data for training, and one-third of the data for testing. The dataset only includes straight sections of the colon. Since the weights of ANNs are randomly generated, and are not guaranteed to converge to a global minimum, 100 ANNs were generated for each optical flow/partitioning method. Using the means and standard deviations as a basis for evaluation, the best ANNs were chosen to perform the estimations.

\section{B. Experimental Results and Discussion}

Figure 5 shows the pose estimation capabilities for each of the optical flow methods using grid-based partitioning and lumen-centered partitioning for 1 DOF robotic actuation along a straight section of the colon. Figure 5(a) shows the mean and the standard deviation of the incremental pose error (i.e., between the output of the ANNs and the groundtruth endoscope motion) for the grid-based partition. The two dense optical flow methods performed similarly, with an average absolute mean of $1.3 \mathrm{~mm} \pm 1.9 \mathrm{~mm}$ error over the moving direction ( $\mathrm{X}$ axis), and outperformed the ANNs resulting from the sparse optical flow methods. The absolute mean errors using these methods spanned to $14.6 \mathrm{~mm}$ using FAST with SURF descriptors. Note in Figs. 5(a) and 6(a), that the translation errors along the $\mathrm{Y}$ and $\mathrm{Z}$ axis are zero, suggesting the ANNs capability to correctly identify the moving direction. Moreover, our orientation errors were zero

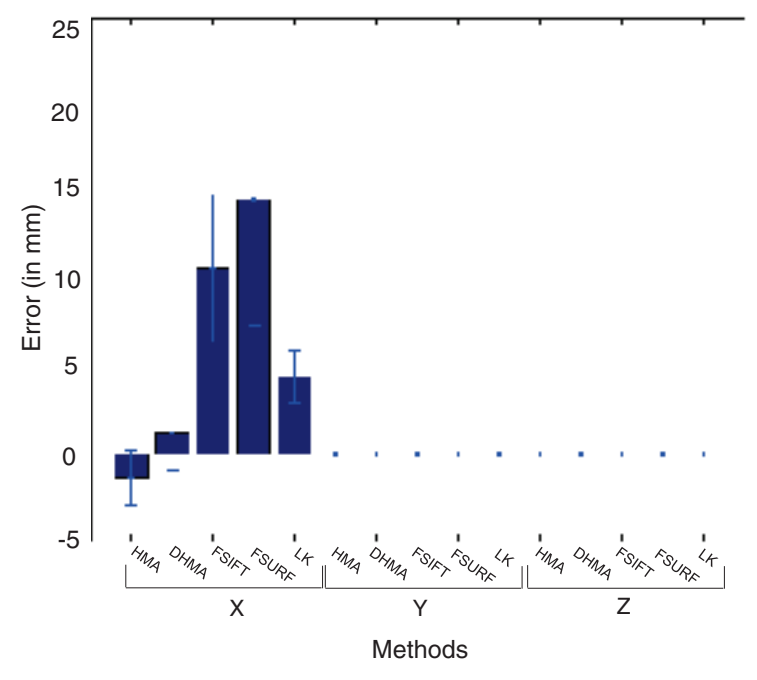

(a)

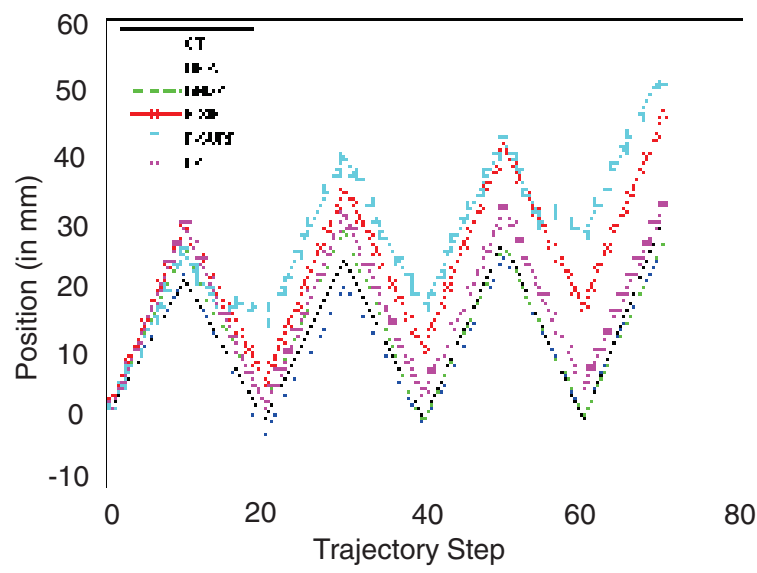

(b)

Fig. 5. Experimental results with grid-based partitioning. (a) Mean and standard deviation of error. (b) Trajectory of endoscope as compared with ground truth of robot encoders.

for both grid and lumen based ANNs, for this reason we decided to do not include the orientation errors in Figs. 5-6, as well as to limit our analysis to only the $\mathrm{X}$ axis.

For each method using the grid-based partition, the integration of the estimated incremental positions along the $\mathrm{X}$ axis are shown in Fig. 5(b) to form the entire estimated trajectory along the forward direction of the endoscope during the trial. The dense optical flow techniques are best able to produce nearly the same trajectory with a final difference of $3.61 \mathrm{~mm}$ over $174 \mathrm{~mm}$. FAST-SURF performed the worst, with a deviation of $19.51 \mathrm{~mm}$ from the intended trajectory.

As for the performance of the optical flow methods using the lumen-centered partition, FAST-SIFT, FAST-SURF, and dHMA all perform similarly, with approximately $4-5 \mathrm{~mm}$ of mean absolute error over the entire test set. However, unlike the grid-based partitioning, the LK method is able to achieve the lowest absolute mean error $(0.31 \mathrm{~mm})$, although 


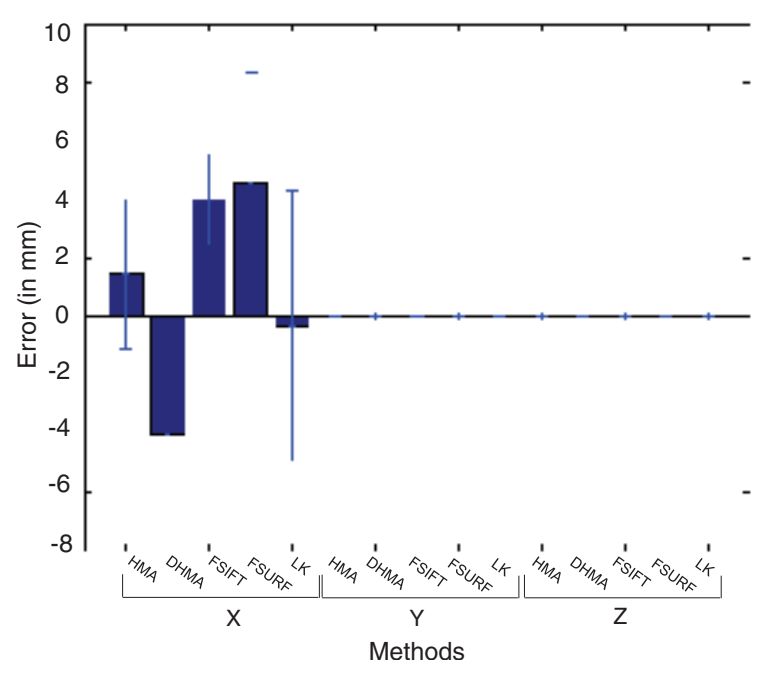

(a)

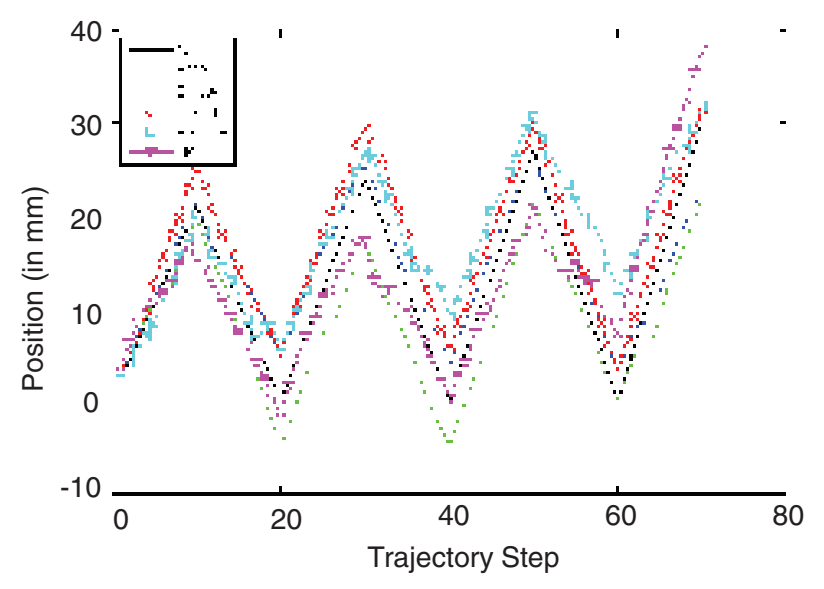

(b)

Fig. 6. Experimental results with lumen-centered partitioning. (a) Mean and standard deviation of error. (b) Trajectory of endoscope as compared with ground truth of robot encoders.

it also possesses the highest standard deviation $( \pm 4.6 \mathrm{~mm})$. On the other hand, HMA results in a slightly higher mean error $(1.45 \mathrm{~mm})$, but the standard deviation is much smaller $( \pm 2.55 \mathrm{~mm})$

Figure 6(b) demonstrates performance of these algorithms over time when the changes in position are integrated to form an entire trajectory. As shown, the mean of each algorithm appears to correspond to an offset from the ground truth data. For example, HMA, with a small mean and standard deviation, is able to closely follow the trajectory until time step 63. FAST-SIFT, FAST-SURF and dHMA exhibit larger offsets during the trajectory, more evident during changes of direction (peaks). Noteworthy is the performance of the LK optical flow, whose sparse nature causes large standard deviation causes unpredictable behavior in the motion estimation, thus resulting in an unpredictable localization estimate.

Overall, the grid-based partition produces two of the neural networks with the lowest error and smallest standard deviation over both partitions; however lumen-centering partitioning appears to standardize the scene, which results in lower error over all of the optical flow methods.

It is important to note that these results are dependent on the training of the neural networks. Although 100 trials were used in order to select the best neural network for each optical flow/partitioning method, a more exhaustive number of trials or averaging the outputs of all these neural networks may represent a more reliable solution for comparing these methods.

\section{CONCLUSiOnS AND Future WORK}

Colorectal cancer affects the lives of millions of people worldwide; although almost always preventable, patients avoid recommended colorectal cancer screening due to fear of discomfort, and the perceived indignity of the procedure. Teleoperable flexible endoscopes are emerging as a novel method to abate these hindrances, but require closed-loop control due to the complexities of the environment of the colon. Pose detection using only the endoscopic camera stream represents a favorable solution to this problem, since it uses only components native to the endoscope, and can be implemented across all teleoperable endoscopic platforms.

The proposed approach estimates the change in pose of the endoscope by extracting the optical flow, the motion between two sequential images. Five methods of calculating the optical flow were compared: LK, FAST-SIFT, FASTSURF optical flow, HMA, and dHMA based on their ubiquity in usage. Additionally, two partitioning methods - grid-based and lumen-centered - were then compared to create single optical flow descriptors for the optical flow in the entire image. To produce a metric measurement of the change in pose, an ANN was trained based on these inputs and the known pose change of the endoscope.

This algorithm was then tested using an endoscope rigidly attached to a robotic arm to mimic the expected movements of a teleoperable flexible endoscope. The experiment was performed on a human colon simulator in order to resemble the endoscopic environment. Using the grid-based partitioning method, the dense optical flow algorithms were the most accurate $(1.3 \mathrm{~mm} \pm 1.9 \mathrm{~mm}$ error; final difference in trajectory $3.61 \mathrm{~mm}$ over $174 \mathrm{~mm})$.

Regarding the lumen-centered partitioning, the LK optical flow resulted in the lowest mean error; however, the imprecision of the algorithm caused large fluctuations in the overall integrated trajectory. HMA provides the best performance with lumen-centered partitioning $(1.45 \mathrm{~mm} \pm 2.55 \mathrm{~mm})$; it has the smallest standard deviation, and is able to closely recreate the trajectory as given by ground truth. Between the grid-based partitioning and the lumen-centered partitioning, the grid-based partition produces the two best performing neural networks as measured by their means and standard deviations; however, the lumen-centered approach appears to standardize the image, resulting in an overall lower average error among all the techniques.

Future work includes investigating other robust, stable features for detecting optical flow in the colon, investigat- 
ing other descriptor for representing the optical flow, and finding the optimal method for mapping the optical flow to the change in pose of the endoscope. Furthermore, more extensive analysis needs to be done to quantify the sensitivity of the ANNs to the size of the training set, as well as the robustness to optical flow ambiguities caused by different motions with similar optical flow. Additionally, in-vivo trials will be performed in order to analyze the performance of the algorithm on human colon tissue. From this, the impact of haustal contractions and small movements of the colon can be assessed. The performance of our algorithm indicates that pose detection via supervised learning of optical flow is a feasible feedback mechanism for implementing closed-loop control of teleoperated flexible endoscopes.

\section{REFERENCES}

[1] F. Haggar and R. Boushey, "Colorectal cancer epidemiology: Incidence, mortality, survival, and risk factors," Clinics in Colon and Rectal Surgery, vol. 22, no. 4, pp. 191-197, 2009.

[2] Fact sheet \# 297: Cancer. World Health Organization (WHO). Last accessed: 1 February 2012. [Online]. Available: www.who.int/mediacentre/factsheets/fs297/en .

[3] P. Valdastri, M. Simi, and R. J. Webster III, "Advanced technologies for gastrointestinal endoscopy," Annual Review of Biomedical Engineering, vol. 14, pp. 397-429, 2012.

[4] Vital Signs Cancer screening, colorectal cancer. Centers for Disease Control and Prevention. Last accessed: 1 February 2012. [Online]. Available: www.cdc.gov/vitalsigns/CancerScreening/indexCC.html .

[5] K. L. Obstein and P. Valdastri, "Advanced endoscopic technologies for colorectal cancer screening." World J Gastroenterol, vol. 19, no. 4, pp. 431-9, 2013.

[6] R. Reilink, S. Stramigioli, and S. Misra, "Three-dimensional pose reconstruction of flexible instruments from endoscopic images," in Proceedings of the 2011 IEEE/RSJ International Conference on Intelligent Robots and Systems, 2011, pp. 2076-2082.

[7] N. van der Stap, R. Reilink, S. Misra, I. Broeders, and R. van der Heijden, "The use of the focus of expansion for automated steering of flexible endoscopes," in 4th IEEE RAS EMBS International Conference on Biomedical Robotics and Biomechatronics (BioRob), 2012, pp. 13 18.

[8] J. Ruiter, E. Rozeboom, M. van der Voort, M. Bonnema, and I. Broeders, "Design and evaluation of robotic steering of a flexible endoscope," in 4th IEEE RAS EMBS International Conference on Biomedical Robotics and Biomechatronics (BioRob), 2012, pp. 761767.

[9] J. Rey, H. Ogata, N. Hosoe, K. Ohtsuka, N. Ogata, K. Ikeda, H. Aihara, I. Pangtay, T. Hibi, S. Kudo, and H. Tajiri, "Blinded nonrandomized comparative study of gastric examination with a magnetically guided capsule endoscope and standard videoendoscope," Gastrointestinal Endoscopy, vol. 75, no. 2, pp. 373-381, 2012.

[10] J. Keller, C. Fibbe, F. Volke, J. Gerber, A. C. Mosse, M. ReimannZawadzki, E. Rabinovitz, P. Layer, D. Schmitt, V. Andresen, U. Rosien, and P. Swain, "Inspection of the human stomach using remote-controlled capsule endoscopy:a feasibility study in healthy volunteers (with videos)," Gastrointestinal Endoscopy, vol. 73, no. 1, pp. 22-28, 2011.

[11] P. Valdastri, G. Ciuti, A. Verbeni, A. Menciassi, P. Dario, A. Arezzo, and M. Morino, "Magnetic air capsule robotic system: proof of concept of a novel approach for painless colonoscopy," Surgical Endoscopy, vol. 26, no. 5, pp. 1238-46, 2011.

[12] X. Wang, M. Meng, and X. Chen, "A locomotion mechanism with external magnetic guidance for active capsule endoscope," in 32nd Annual International Conference of the IEEE Engineering in Medicine and Biology Society, 2010, pp. 4375-4378.

[13] G. Khan and D. Gillies, "Vision based navigation system for an endoscope," Image and Vision Computing, vol. 14, no. 10, pp. 763 - 772, 1996.

[14] X. Zabulis, A. Argyros, and D. Tsakiris, "Lumen detection for capsule endoscopy," in Proceedings of the 2008 IEEE/RSJ International Conference on Intelligent Robots and Systems, 2008, pp. 3921-3926.
[15] S. Krishnan, C. Tan, and C. Chan, "Closed-boundary extraction of large intestinal lumen," in Proceedings of the 16th Annual International Conference of the IEEE Engineering in Medicine and Biology Society, vol. 1, 1994, pp. 610-611.

[16] H. Tian, T. Srikanthan, and K. Asari, "Automatic segmentation algorithms for the extraction of lumen region and boundary from endoscopic images," Medical and Biological Engineering and Computing, vol. 39, no. 1, pp. 8-14, 2001.

[17] S. Xia, S. Krishnan, M. Tjoa, and P. Goh, "A novel methodology for extraction colon's lumen from colonoscopic images," Journal of Systemics, Cybernetics and Informatics, vol. 1, no. 2, pp. 7-12, 2003.

[18] J. Bulat, K. Duda, M. Duplaga, R. Fraczek, A. Skalski, M. Socha, P. Turcza, and T. Zielinski, "Data processing tasks in wireless GI endoscopy: Image-based capsule localization \& navigation and video compression," in Proceedings of the 29th Annual International Conference of the IEEE Engineering in Medicine and Biology Society, 2007, pp. 2815-2818.

[19] R. Hartley and A. Zisserman, Multiple View Geometry in Computer Vision, 2nd ed. New York, NY, USA: Cambridge University Press, 2003.

[20] J. Liu, T. Yoo, K. Sabramanian, and R. V. Uitert, "A stable opticflow based method for tracking colonoscopy images," in Proceedings of the 2008 IEEE Computer Society Conference on Computer Vision and Pattern Recognition Workshops, June 2008, pp. 1-8.

[21] J. Liu, K. Subramanian, and T. Yoo, "Towards designing an opticalflow based colonoscopy tracking algorithm: a comparative study," in Proc. SPIE, 2013, pp. 867 103-867 107.

[22] C. Bell, P. Valdastri, and K. Obstein, "Image partitioning and illumination in image-based pose detection for teleoperated flexible endoscopes," Artificial Intelligence in Medicine, vol. 59, no. 3, pp. 185-196, 2013.

[23] B. D. Lucas and T. Kanade, "An iterative image registration technique with an application to stereo vision," in Proceedings of the 7th International Joint Conference on Artificial Intelligence (IJCAI), 1981, pp. 674-679.

[24] J. Shi and C. Tomasi, "Good features to track," in Proceedings of the IEEE Conference on Computer Vision and Pattern Recognition, 1994, pp. 593-600.

[25] G. Bradski, "The OpenCV Library," Dr. Dobb's Journal of Software Tools, 2000.

[26] D. G. Lowe, "Distinctive image features from scale-invariant keypoints," Int. J. Comput. Vision, vol. 60, no. 2, pp. 91-110, November 2004.

[27] K. Mikolajczyk and C. Schmid, "A performance evaluation of local descriptors," IEEE Transactions on Pattern Analysis and Machine Intelligence, vol. 27, no. 10, pp. 1615-1630, 2005.

[28] E. Rosten and T. Drummond, "Machine learning for high-speed corner detection," in Proceedings of the 9th European conference on Computer Vision, 2006, pp. 430-443.

[29] G. Puerto-Souza and G. Mariottini, "A fast and accurate featurematching algorithm for minimally-invasive endoscopic images," IEEE Transactions on Medical Imaging, vol. 32, no. 7, pp. 1201-1214, July 2013.

[30] - "HMA feature-matching toolbox,"

[Web:] http://ranger.uta.edu/\% 7 egianluca/feature $\% 5$ matching

[31] - "Wide-baseline dense feature matching for endoscopic images," in 6th Pacific Rim Symposium in Advances in Image and Video Technology, PSIVT 2013, November 2013, in press.

[32] C. Rasmussen and C. Williams, Gaussian processes for machine learning. MIT press Cambridge, MA, 2006, vol. 1.

[33] L. Sherwood, Human Physiology: From Cells to Systems, 6th ed. Thomson Brooks/Cole, Pacific Grove, CA, USA, 2007.

[34] G. Cybenko, "Approximation by superpositions of a sigmoidal function," Mathematics of Control, Signals, and Systems (MCSS), vol. 2, no. 4, pp. 303-314, 1989.

[35] K. Levenberg, "A method for the solution of certain nonlinear problems in least squares," Quarterly of Applied Mathematics, vol. 2, pp. 164-168, 1944.

[36] D. Marquardt, "An algorithm for least-squares estimation of nonlinear parameters," SIAM Journal on Applied Mathematics, vol. 11, no. 2, pp. 431-441, 1963

[37] R. Hecht-Nielsen, "Kolmogorov's mapping neural network existence theorem," in Proceedings of the IEEE First Annual International Conference on Neural Networks, S. Grossberg, Ed., vol. 3, 1987, pp. 11-14. 